\title{
Estudio de plantas medicinales usadas en la comunidad indígena Tikuna del alto Amazonas, Macedonia.
}

\author{
Ronald Fernando Quintana Arias ${ }^{1}$ \\ ${ }^{1}$ Licenciado en Biología, MSc. (Ca) en Desarrollo Sustentable y Gestión Ambiental. \\ Universidad Distrital Francisco José de Caldas de Bogotá (Colombia)
}

Correspondencia: ron902102004@gmail.com

Recibido: 17/08/2012 Aceptado:12/12/2012

\begin{abstract}
Resumen
Entre Febrero del 2008 y Octubre del 2009, se realizó una investigación de plantas asociadas a las enfermedades más frecuentes en la Comunidad TIKUNA de Macedonia ubicada a $57 \mathrm{~km}$ de Leticia. Para realizar este estudio se aplicaron tres tipos de encuestas basadas en las "TRAMIL". A a partir de estas, se establece como resultado el reporte de 115 plantas de valor de uso medicinal dentro de la Comunidad, y 308 remedios aplicados para la cura de diferentes enfermedades. Se presentó un listado que incluye: 109 géneros y 99 especies. Para cada una de las plantas medicinales se reporta el nombre vernáculo, nombre Tikuna, número de colección, familia taxonómica, usos tradicionales referidos, parte usada, propiedad medicinal, forma de preparación, administración, si la preparación en caso de ser combinada se hace con una planta o animal y si debe tener "dieta" previamente en el SINCHI ${ }^{3}$ de Leticia, se calculó el nivel de uso significativo TRAMIL, valor de uso general, fitoetnoaloctoneidad y Fitoetnoendemicidad.
\end{abstract}

Se concluye que las familias Arecaceae, Bignoniaceae y Rubiaceae y especies como el Yarumo (Cecropia sciadophylla) el Carambolo (Averrhoa carambola), la uńa de gato (Uncaria tomentosa), el Acapu (Minquartia guianensis), la Lancetilla (Alternanthera brasiliana), y el Amacizo (Erythrina fusca) tienen un gran valor cultural dentro de la Comunidad. Asimismo, que el uso y aplicación de diferentes plantas que alivien dolencias es compartido por las etnias del Trapecio, pero la validez u orden de importancia de las plantas depende de la cosmología y cosmogonía particular de cada comunidad.

Palabras clave: Amazonas, enfermedades, etnobotánica, plantas medicinales, Tikunas.

2 Hace alusión a un sacrificio personal, generalmente asociado con la comida o el sexo.

3 Instituto Amazónico de Investigaciones científicas. 


\title{
Study of medicinal plants used by the Tikunas indigenous community of the upper Amazon (Macedonia)
}

\begin{abstract}
A study of the plants associated with most common diseases in the TIKUNA community of Macedonia (located $57 \mathrm{~km}$ from Leticia, Colombia) were carried out from February 2008 to October 2009. Three types of surveys based on the "TRAMIL were applied. Total 115 plants with medicinal use and 308 remedies applied to cure various diseases were determined comprising 109 genera and 99 species. For each plant the vernacular name, Tikunas name, collection number, taxonomic family, traditional uses, part used, medicinal properties, method of preparation and administration, how was prepared (combined with a plant or animal) and whether it should be "diet" or not were documented. Each plant was documented: photographed on the field, collected and identified previously in SINCHI Leticia. The level of TRAMIL significant use value was calculated as well as phyto-endemicity and fitoetnoaloctoneidad.
\end{abstract}

Families Arecaceae, Bignoniaceae and species such as the Rubiaceae and Yarumo (Cecropia sciadophylla) the Carambolo (carambola), cat's claw (Uncaria tomentosa), the Acapu (Minquartia guianensis), the Lancetilla (Alternanthera brasiliana) and the Amacizo (Erythrina fusca) have great cultural value within the community. Additionally the use and application of different plants to relieve ailments were found shared by trapezium ethnic, although the validity or importance of plants depends on the cosmology and cosmogony of each community.

Keywords: Amazon, diseases, ethno-botany, medicinal plants, Tikuna.

\section{Introducción}

El estudio de la Amazonia ha sido un tema de gran importancia por su connotación biológica y cultural, en donde los estudios etnobotánicos han tenido gran importancia por su intento de generar un acercamiento hacia el conocimiento de la potencialidad de la diversidad biológica del Amazonas (1-4).

De esta manera las comunidades ribereñas de la Amazonia han tenido un gran número de estudios de valor de uso de las plantas (5) cuyos resultados y alcances positivos y negativos han sido discutidos por varios autores Mantilla et al, 2007 (6); Arias y Prieto, 2005 (7); Gonzales, 1997 (8); Rachel-Dolmatoff, 1997 (9); GermosénRobineau,1995 (10); Philips,1996 (11); Philips \& Gentri, 1993(11); Van Der Hammen, 1991(12);
Shultes \& Raffauf ,1990 (13); Prance et al, 1987 (14); Balee, 1986 (15); Palacios, 1986 (16); Glemboski, 1983 (17), así como el SINCHI en sus publicaciones del acta Amazónica.

La identificación taxonómica de plantas asociadas a las enfermedades han generado conflictos y faltas a acuerdos internacionales en donde se desconocen los dueños del conocimiento, dándole relevancia más a la tecnología y no a la cultura -Declaración de Chiang Mai; WHA30.49 de 1977; Resolución 5078 de 1992; Resolución V. 1993; Resolución WHA51.24- (18). Debido a esto, los proyectos etnobotánicos deben ser una tarea participativa de la misma connotación y orden de importancia a los actores involucrados para generar procesos de perpetuidad cultural, rescatando el conocimiento local (19-20). 
Este artículo se basa en estudio descriptivo con un enfoque mixto, en donde lo cualitativo es conformado por los usos reportados por las etnias de la comunidad y lo cuantitativo se basa en el número de plantas identificadas. Se buscó establecer un canal semiótico en dos vías estableciendo: nombres indígenas y vernáculos de las plantas, familias taxonómicas más importantes, reporte de usos para las familias taxonómicas y familias taxonómicas más importantes para la comunidad. Se colectaron con base a la metodología del Grupo Tramil y el SINCHI, 115 plantas medicinales, de las cuales se identificaron 99 especies, 109 géneros, 115 familias con la asesoría de Juan Carlos Arias del instituto geográfico de investigaciones científicas (SINCHI). Este número de plantas reportadas es compartido por otros estudios etnobotabicos de la zona: Bejarano, 2003 (21) con una lista de 129 plantas medicinales, Palacios, 1986 (22) con una lista de 85 plantas medicinales, Glenboski, 1983 (17) con una lista de 83 plantas medicinales, La Rotta, 1982 y 1983 (23-24) con una lista de 33 y 154 plantas respectivamente.

\section{Materiales y métodos}

\section{1. Área de estudio}

Macedona es una comunidad ribereña del Amazonas colombiano ubicada entre las comunidades de Mocagua y El Vergel a 57 kilómetros de Leticia; pertenece a la zona de amortiguación Parque $\mathrm{Na}$ tural Nacional Amacayacu y constituye uno de los asentamientos más grandes del río, aproximadamente con 700 habitantes y 100 familias (25-26).

\section{Descripción metodológica}

Para el trabajo en comunidad se tuvo como referente la Investigación Acción Participante (I.A.P), cambiando la visión de sujeto-objeto buscando provocar una reacción reflexiva en la Comunidad (27). De esta manera el proceso metodológico se dividió en tres fases: campo, laboratorio y análisis.

Para la primera fase del estudio se concertó la propuesta de investigación con el curaca y posteriormente se realizó una concertación con la comunidad.
Una vez aprobado el proyecto se aplicó un enfoque metodológico basado en el "Consenso de Informantes" (11, 28-29), el cual permite realizar una evaluación cuantitativa de la importancia del uso de las plantas dentro de la comunidad. Con este fin se realizaron tres encuestas basadas en el estudio etnobotánico de Moreno, 2005 (30) y en las de nivel de uso significativo del grupo de investigación de estudios en el neotrópico "TRAMIL". Esto permitió encontrar específicamente plantas con actividad farmacológica, en contraposición a otras metodologías como las seleccionadas al azar o por criterios quimiotaxonómicos (31-33).

La primera encuesta se aplicó a los jefes de hogar con el fin de identificar a los sabedores. Posteriormente, se realizó una encuesta a los sabedores con el fin de elaborar un inventario de las especies vegetales reportadas como de uso medicinal. Luego de este paso se realizó la recolección de las muestras vegetales con los sabedores, donde las plantas fueron identificadas por ellos (nombres locales) en su sitio de recolección. Paso siguiente, se prepararon los especímenes vegetales para su identificación, realizando la traducción de los nombres de las plantas a lengua Tikuna con el profesor de etnolingüística dentro de la comunidad, Antero León Macedo. En el procesamiento de las muestras se siguieron los protocolos del Centro de Investigaciones Científicas (SINCHI). El secado, la preparación y la identificación fueron realizados en el herbario del Instituto de Investigaciones Amazónicas (COAH), contando con la asesoría del Amazonólogo Juan Carlos Arias, investigador de flora y fauna del instituto.

Para la fase de análisis se generó un diagnóstico de los problemas de salud más frecuentes en la comunidad, utilizando los datos proporcionados por los sabedores y los jefes de hogar, a través de la información etnofarmacológica sobre las plantas utilizadas. Para ello se calcularon índices de: nivel de uso significativo de carácter cuantitativo (UST), de uso (IVUs), fitoetnoaloctoneidad (IFEA) y fitoetnoendemicidad (IFEE). 
Nivel de usos significativo (UST). Este índice expresa que los usos medicinales citados con una frecuencia superior o igual al $20 \%$, por las personas encuestadas que usan plantas como primer recurso para un determinado problema de salud, pueden considerarse significativos desde el punto de vista de su aceptación cultural. Por lo tanto, merecen su evaluación y validación científica (34). El UST se calcula dividiendo el número de citaciones de uso para cada especie (s), entre el número de informantes encuestados, se propone la siguiente ecuación:

UST $=\frac{\text { Uso Especie }(s)}{\text { nis }} * 100$

$\mathrm{UST}=\frac{\text { Uso Especie }(\mathrm{s})}{\text { nis }} * 100$

\section{Donde:}

Uso Especie $(s)$ = número de citaciones para cada especie.

nis $=$ número de informantes encuestados.

Indice o valor de uso (IVUs). Este valor expresa la importancia o valor cultural de una especie determinada para todos los informantes entrevistados (29). Para estimar el índice de valor de uso general de cada especie para todos los informantes (IVUs), se utilizó la fórmula:

IVUs $=\frac{\sum_{\mathrm{i}} \mathrm{UVis}}{\mathrm{ns}} \mathrm{IVUs}=\frac{\sum_{\mathrm{i}} \mathrm{UVis}}{\mathrm{ns}}$

\section{Donde:}

UVis = Número de usos mencionados por cada informante (i) para cada especie (s).

ns $=$ Número de informantes entrevistados.

Fitoetnoaloctoneidad (IFEA). Este índice informa sobre el grado de dependencia de la comunidad de Macedonia con otras comunidades, el nivel en que el medio no ofrece soluciones a un determinado grado de requerimientos y el carácter de la población (si es dada al intercambio material y cultural a las comunidades y al comercio) (35). Se refiere a las plantas que son usadas en la zona pero no crecen, ni se cultivan de forma espontánea en la misma. Para estimar la Fitoetnoaloctoneidad, se utilizó la fórmula:

$\mathrm{IFEA}=\frac{N S A U \times 100}{\mathrm{NTEE}} \mathrm{IFEA}=\frac{N S A U \times 100}{\mathrm{NTEE}}$

\section{Donde:}

NSAU = Número de plantas alóctonas empleadas.

NTEE $=$ Número total de plantas con valor de uso medicinal usadas en la comunidad

Fitoetnoendemicidad (IFEE). Este cálculo se realiza a partir de las plantas que existen en otras comunidades pero solo se utilizan en la comunidad. Para estimar la Fitoetnoendemicidad, se utilizó la fórmula:

$\mathrm{IFEE}=\frac{N F E N \times 100}{\mathrm{NTEU}} \mathrm{IFEE}=\frac{N F E N \mathrm{x} 100}{\mathrm{NTEU}}$

\section{Donde:}

NFEN = Número de fitoetnoendemismos.

$\mathrm{NETEU}=$ Número total de plantas con valor de uso medicinal usadas en la comunidad.

\section{Resultados}

A partir de la recolección de las muestras vegetales y el conteo total de especies se determinó que la familia Solanaceae es la más representativa de Macedonia, encontrándose 7 especies, Tabla 1.

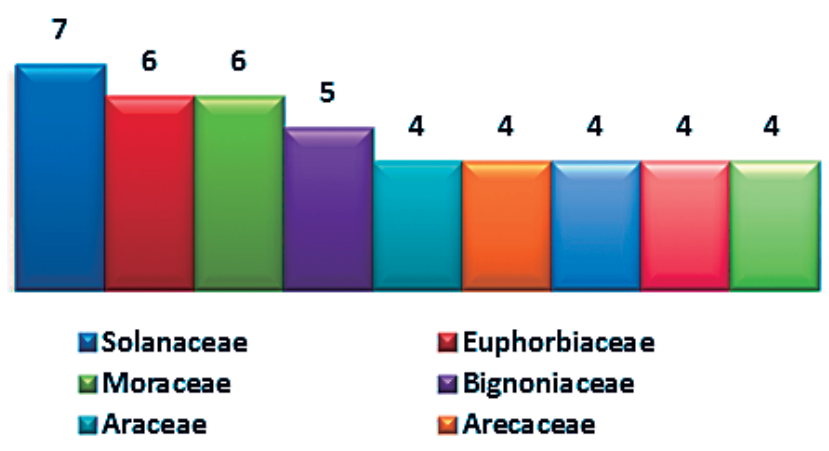

Figura 1. Familias Vegetales más representativas en Macedonia. 
Con base en los reportes del puesto del salud de la comunidad y las encuestas aplicadas a la población, se encontró que el uso de las plantas medicinales para tratar las enfermedades gastrointestinales es el más recurrente en la comunidad con 23 reportes, seguido del tratamiento de enfermedades dérmicas con 21 reportes y en menor proporción, los usos abortivo y anticonceptivo con un solo reporte.
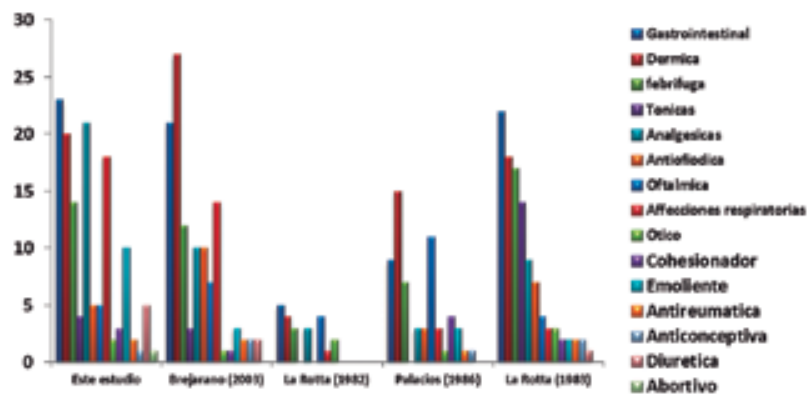

Figura 2. Comparación de resultados asociados a los usos de las plantas medicinales en diferentes estudios en la Amazonia

Las encuestas aplicadas a los sabedores revelaron que el $96,35 \%$ no hacen dieta antes de aplicar el tratamiento medicinal tradicional. Adicionalmente, la mayoría de los encuestados afirmaron administrar oralmente los remedios que elaboran a partir de las plantas, figura 3, de las cuales se emplea principalmente las hojas para la preparación de los mismos, figura 4. Adicionalmente, se estableció que en el $84,31 \%$ de los tratamientos se deben combinar especies de plantas para que sean efectivos contra una enfermedad específica, figura 5.

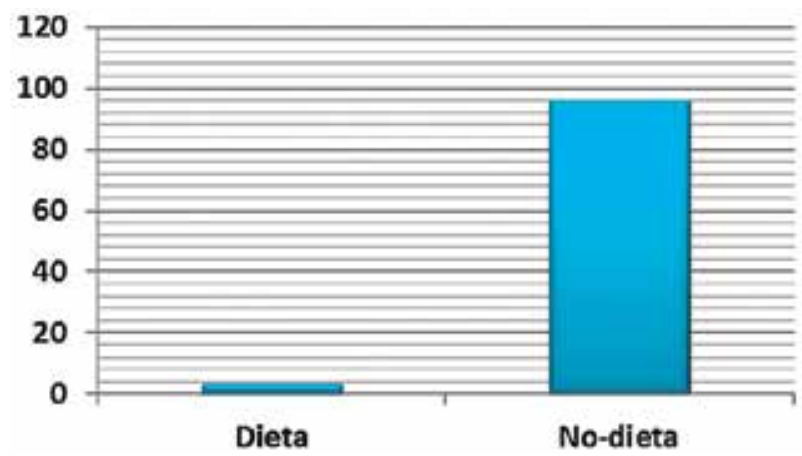

Figura 3. Porcentaje de privaciones personales al momento del tratamiento medicinal tradición.

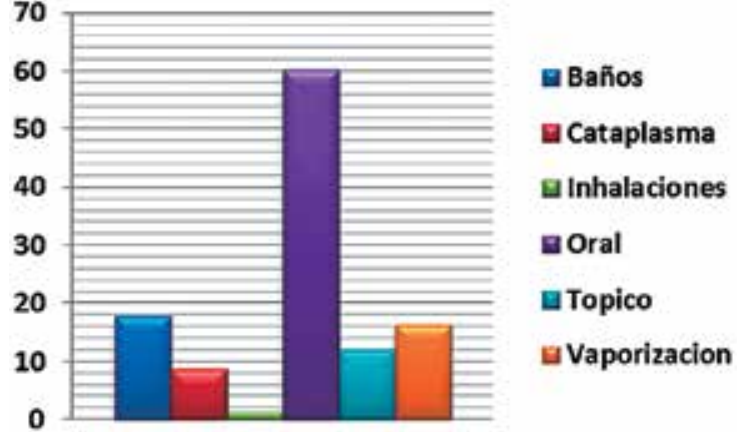

Figura 4. Porcentaje de formas de administración para el tratamiento de enfermedades

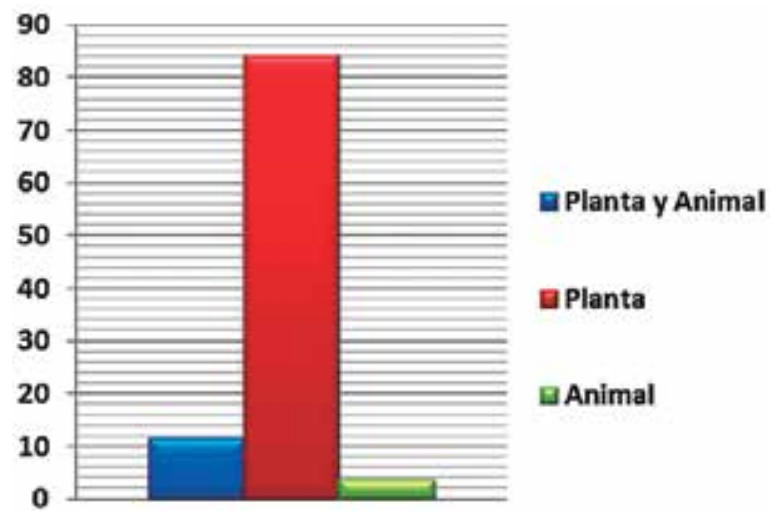

Figura 5. Combinaciones usadas en la preparación de remedios
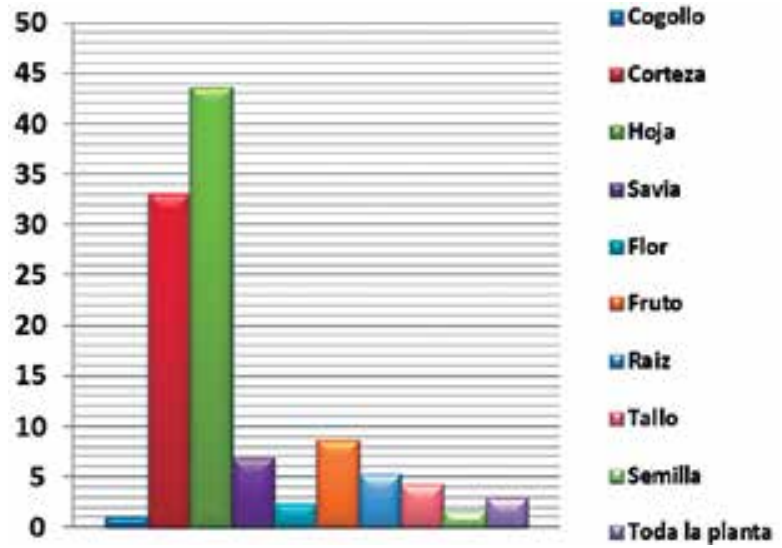

Figura 6. Porcentaje de partes de las plantas utilizadas para preparar remedios.

En la tablas 1 y 2 se reportaron para cada especie la clasificación taxonómica, los usos medicinales, las formas y combinaciones para la preparación, la vía de administración, el nivel de uso significativo, la dieta y Número de citaciones y los índices de valor de uso (IVUs), Fitoetnoaloctoneidad (IFEA) y Fitoetnoendemicidad (IFEE). 


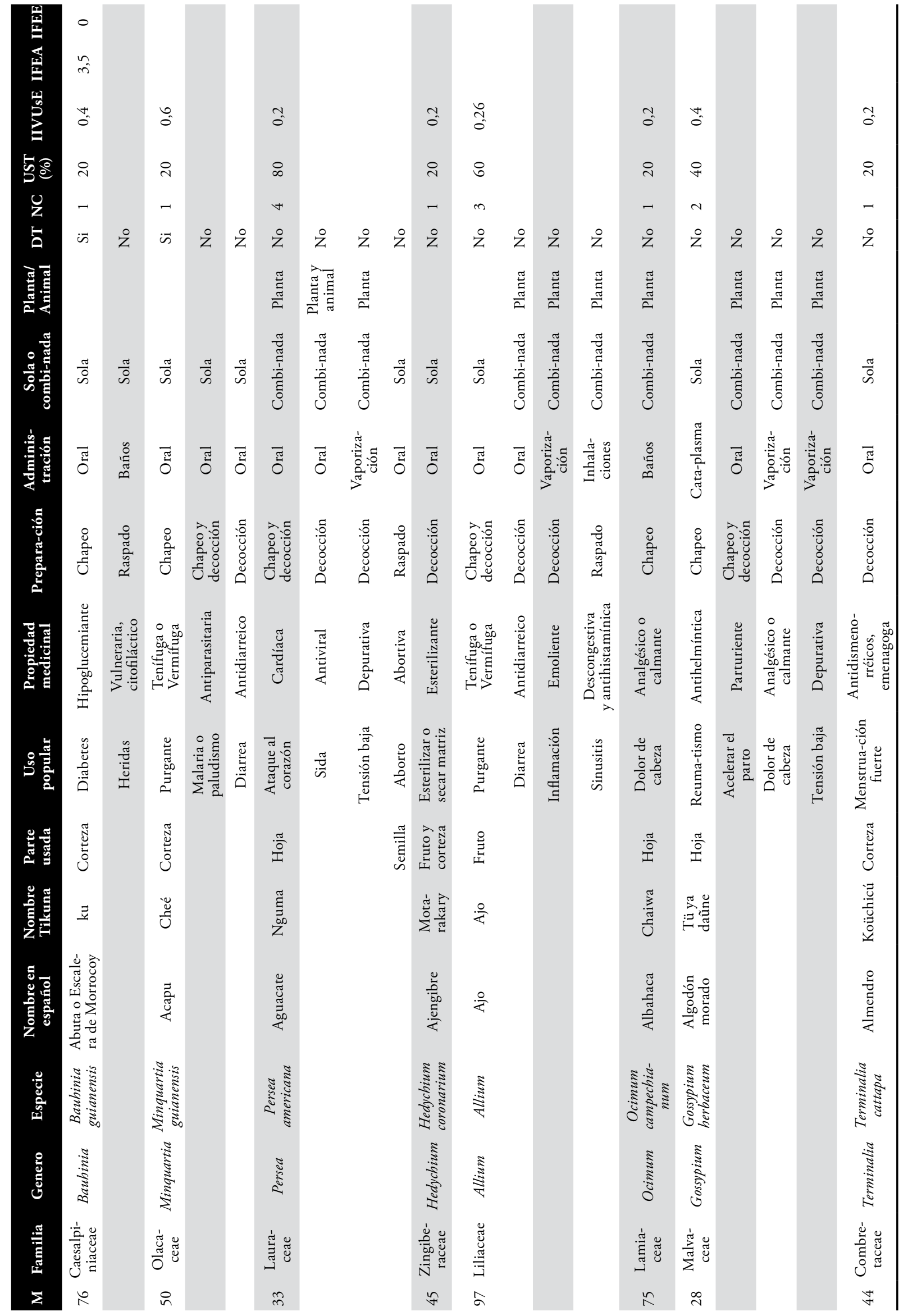




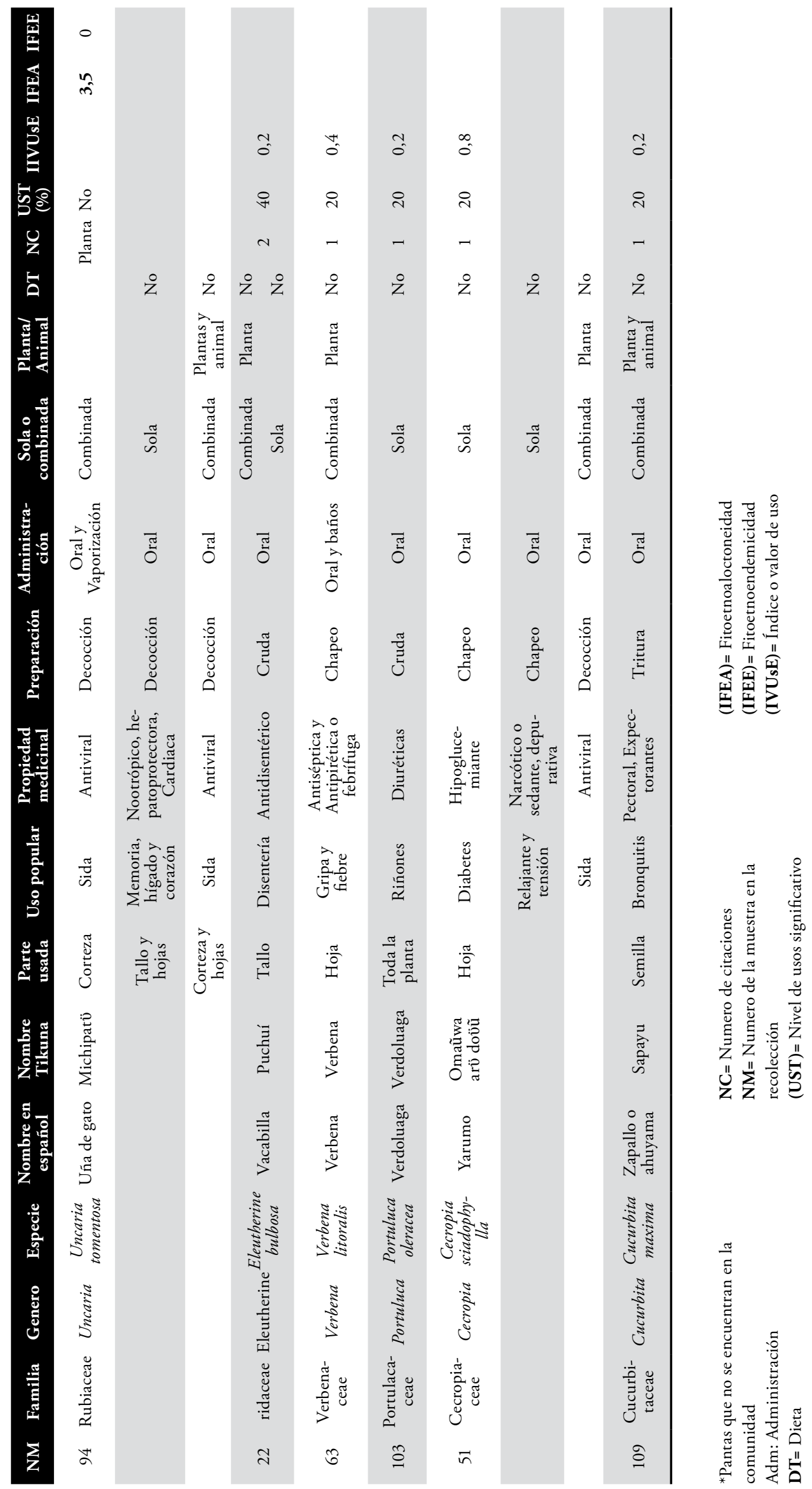




\section{Discusión}

Análisis Florístico. Se colectaron 115 plantas medicinales, de las cuales se identificaron 99 especies, 109 géneros, 115 familias. El número de Plantas medicinales (115) encontradas en este estudio, están por debajo del rango de estudios etnobotánicos realizados en la amazonia no colombiana; el rango máximo reportado para los Ashur de ecuador con 245 plantas y el mínimo para los Káapor con 110 plantas $(4,36-38)$.

Pese a esto, el número de plantas medicinales reportadas en el presente artículo, a nivel de la Amazonía colombiana es compartido por otros estudios: Bejarano, 2003 (21) con una lista de 129 plantas medicinales, Palacios ,1986 (22) con una lista de 85 plantas medicinales, Glenboski, 1983 (17) con una lista de 83 plantas medicinales, La Rotta, 1982 y 1983 (23-24) con una lista de 33 y 154 plantas respectivamente. El hecho que para la Amazonía colombiana, se registren bajos niveles de reportes en comparación con los de otros países, se puede explicar desde la divergencia en el grado de los procesos de aculturización, deculturización e integración (39) que se presenta entre los Tikuna Brasileños y colombianos.

Los estudios que han hablado del grado de aculturización en la Amazonía colombiana (40); Prance, 1972 en Estrella 1995 (41), concluyen que la exposición de una minoría étnica a la Cultura Occidental desencadena en su primera fase un incremento en el número de las plantas medicinales seguido por un descenso en el que solo permanecen las cultivadas. Con referencia al trabajo de campo se encuentra que el lugar de obtención de las plantas tiene un mayor porcentaje para la huerta o jardín (80\%) lo que evidencia un alto nivel de aculturización de las prácticas tradicionales curativas.

De las 60 familias vegetales encontradas en este estudio, Tablas 1-2, las más representativas son la Solanaceae (7 especies) Euphorbiaceae (6 especies), Moraceae (6 especies), Bignoniaceae (5 especies) y Araceae, Arecaceae, Asteraceae, Caesalpiniaceae y
Rubiaceae (cada una con 4 especies), Figura 1. El hecho de que en estudios realizados en la Amazonía las familias Arecaceae, Bignoniaceae y Rubiaceae sean las más representativas $(4,16,21)$, sirve como un indicativo de su importancia en cuanto a la riqueza y diversidad en la selva amazónica.

Análisis de uso. Al comparar estudios de uso medicinal en comunidades indígenas, figura 2 , sobresalen las enfermedades más comunes para la comunidad como: gastrointestinales (antiácido, anti diarreico, estomacal, laxante), analgésicas, afecciones respiratorias (tuberculosis, tos, bronquitis, resfrío, neumonía, gripa) y febrífugas. Estas enfermedades ocuparon el primero, segundo, cuarto y quinto lugar entre el total de categorías encontradas. En este estudio, al igual que en el de La Rotta, 1982,1983 (23-24) y Bejarano, 2003(21), estas especies se citan como las categorías más importantes

Cabe resaltar que se registra un alto porcentaje de categoría de uso a las enfermedades dérmicas (escabicidas, vulneraria, dermatológica) y óticas, por lo que el hecho de que estas enfermedades tengan una menor frecuencia de consulta en el puesto de salud de la comunidad, puede atribuirse a que es que estas son las que menor riesgo implican y afectan en menor medida el desarrollo de actividades cotidianas. Así mismo, pueden estar indicando la eficiencia de los remedios que aplica la gente para tratar este tipo de enfermedades, contrario a lo que puede suceder con las otras. También se resalta el hecho que el aborto sea considerado como un uso medicinal de una planta, esto en según Quintana, 2009 (42) se debe a un vestigio del tradicionalismo Tikuna en donde "el ser humano solo se considera humano hasta el momento de nacer" (Entrevista con Buitrago, 2009).

Al analizar la dieta (porcentaje de privaciones personales al momento del tratamiento medicinal tradicional), figura 3, se enmarcan un cuerpo de creencias y privaciones (abstinencia sexual, no consumir alcohol, alimentos salados ni dulces) que de no cumplirse afectan la cura de la enfermedad. 
En este sentido, el efecto de la deculturización dentro de las prácticas tradicionales en la comunidad de Macedonia es mayor a nivel ideológico que práctico, ya que la pérdida de reconocimiento de sus propias costumbres en la forma de colectar las plantas, más que en la acción de recolección en sí, ha generado un desplazamiento de las explicaciones a enfermedades, desde el saber espiritual tradicional a un "nuevo saber espiritual" visto desde el evangelio, que altera la relación tradicional que estos pueblos establecen con la naturaleza.

Este cambio estructural de carácter religioso, ha condicionado las relaciones de la comunidad con el medio ambiente. Esto se evidencia en la figura 3 , en donde se encuentra en una baja proporción el sentido de sacrifico al momento de preparar o tomar algún remedio en la comunidad. Las categorías de uso más comunes, figura 2 , determinan a su vez las aplicaciones más frecuentes, figura 4, por lo que los tratamientos orales, bańos y vaporizaciones son los tratamientos más comunes para las afecciones que más aquejan a la comunidad. Así la forma de aplicación de la planta, depende de la naturaleza de la enfermedad $(17,43)$.

Al realizar el cálculo de los remedios de preparación combinada y el grado de asociaciones de las plantas o animales para preparar algún tipo de remedio dentro de la comunidad, figura 5, se evidencia que en la comunidad la asociación entre dos especies de plantas es mayor que la de una o dos especies de plantas con partes de animales, lo que legitimiza este trabajo como de tipo etnobotánico y no etnobiológico.

La preparación de remedios tradicionales en Macedonia, figura 6 , indica la tendencia a usar todas las partes de la planta en diferentes proporciones dependiendo de su categoría de uso y forma de aplicación. El uso de la corteza está delimitado por el sitio donde salga o se oculta el sol, esto puede estar relacionado con la figura de Yoi e Ipi (cosmovisión Tikuna) o Caín y Abel (cosmovisión Cristiana). Esto ratifica la importancia de la concepción de los "Triksters" a nivel cultural, ya que enmarca el equilibrio cósmico que se da desde la percepción del "bien" y del "mal", y su posible relación en la efectividad o no de algún remedio dentro de la comunidad.

De acuerdo a la información suministrada por los sabedores, a pesar de que existe un conocimiento sobre el grado de toxicidad de diferentes plantas, la elección de las cantidades o dosificación se ciñen a preceptos culturales, donde no hay una dosificación precisa para la preparación de remedios. Este tema es motivo de preocupación ya que puede dar lugar a intoxicaciones o a efectos secundarios inesperados, por lo que se hace necesario que a partir del "sincretismo" se generen canales de comunicación entre la medicina facultativa y tradicional para clarificar y fortalecer la farmacopea local.

No obstante, este sincretismo podría generar un paradigma pues el conocimiento tradicional va más allá del ensayo y del error y es capaz de adaptarse a cambios, sin la necesidad de intervención de otros sistemas médicos. Esto se pudo evidenciar con los reportes en este estudio sobre tratamientos antimalaricos con Chachajo (Mansoa hymenaea) y por el hecho que la malaria no es una enfermedad endémica del Amazonas. A pesar que ésta ingresó a la región hace casi un siglo, fue controlada por la propiedad antimalarica de la planta, que posiblemente se descubrió combatiendo la fiebre (uno de los principales síntomas de la enfermedad).

\section{Análisis numérico de los datos}

Nivel de usos significativo (UST). Dado que se realizó un filtro a través de los líderes de la comunidad para trabajar con los sabedores reconocidos, en este índice se considera que todas las plantas reportadas son significativas desde el punto de vista de su aceptación cultural.

Índice o valor de uso (IVUs). las especies vegetales de valor de uso medicinal con mayor valor cultural 
dentro de la Comunidad son: el Yarumo (Cecropia sciadophylla) y el Carambolo (Averrhoa carambola) cada uno con un valor de uso de 0.8 (80\%), la uña de gato (Uncaria tomentosa), el Acapu (Minquartia guianensis), Lancetilla (Alternanthera brasiliana), con índices de uso de $0.6(60 \%)$ y el Amacizo (Erythrina fusca) con un índice de 0.5 (50\%).

Fitoetnoaloctoneidad (IFEA). se determinó que el grado de dependencia de Macedonia con otras comunidades es muy bajo (3.5), pero hay unas posibles explicaciones al hecho que las plantas de: Ayahuasca (Banisteriposis caapi), Tabaco (Nicotinana tabacun l), Palo de sangre (Brosimun rubescens) y Sangre de grado (Croton lechleri) no se encuentren en la zona.

Macedonia es una comunidad evangélica y esta religión ha vetado el uso de las dos primeras plantas por su valor espiritual tradicional, por lo que ya no se siembra ni se encuentra de manera silvestre en la comunidad. Esto demuestra el efecto de la religión en los procesos mencionados por Ullan de la Rosa, 2000 (39) y como esta afecta la "agroecológica sociocultural" (enteógenos y alucinógenos).

El palo de sangre es una especie de valor de uso económico, ya que se usa para la realización de artesanías dentro de la Comunidad, es una especie que se encuentra casi extinta dado que por lo expresado por los propios indígenas, desconocen el árbol Joven y no saben cómo sembrarlo, en torno a esto la Universidad Distrital de Bogotá y la Universidad Nacional de Colombia han hecho propuestas para la reforestación del palo de sangre en la zona, pero los indígenas aún dudan que los árboles que se han sembrado en la zona sean el palo de sangre.

La sangre de grado es un árbol propio de la alta peruana, su uso y aplicación se debe al contacto que tiene la Comunidad con "cacharreros" peruanos (el termino cacharrero se refiere a comerciantes que venden o canjean juguetes, plásticos, medicinas y en algunos casos animales exóticos). Pese a todo lo anterior, se puede decir que el medio ofrece soluciones a las principales afecciones que sufre la comunidad y que la población es dada al intercambio material y cultural con las comunidades a través del comercio.

Fitoetnoendemicidad (IFEE). Este cálculo se realiza a partir de las plantas que existen en otras comunidades pero solo se utilizan en Macedonia. Se sabe que por este estudio y por los de La Rotta, 1982 \& 1983 (23-24), Palacios, 1986 (22) y Bejarano, 2003 (21) que las enfermedades más comunes en la Amazonía son las gastrointestinales y febrífugas, por lo que el uso y aplicación de diferentes plantas que alivien estas dolencias es compartido por las etnias del Trapecio Amazónico. No obstante, la validez y orden de importancia de estas plantas depende de la cosmología y cosmogonía particular de cada comunidad. A partir del resultado de este índice (0), se concluye que Macedonia no tiene un nivel de aislamiento y sí un alto grado de intercambio cultural. Por lo que la población, por razones del medio no tiene necesidades biológicas o culturales distintas a las de otros grupos humanos en la Amazonía, (35).

Los resultados de todos los instrumentos empleados anteriormente indican un establecimiento de usos medicinal, dado por la relación que se genera entre las etnias y el entorno donde viven. Esto equipara la concepción y el manejo del territorio a una definición de medio ambiente, que incluye la concepción simbólica del paisaje, estableciéndose relaciones que conforman la unidad básica de la interacción con el mundo. Ejemplo de estas relaciones las hace Van der Hammen (1992) territorio-maloca y Zuluaga $(44,45)$ salud-naturaleza y cultura.

\section{Conclusiones}

- Para propiciar una revalorización de la cultura indígena, se debe superar el estereotipo superficial del mismo, ya que este cambio genera actitudes de valor sobre la cultura e importancia de su conservación y trasmisión, tanto a la sociedad nacional como entre los propios indígenas. 
- El hecho de que en este estudio y otros realizados en la Amazonia las familias Arecaceae, Bignoniaceae y Rubiaceae sean las más representativas $(4,21,22)$, sirve como un indicativo de su importancia en cuanto a la riqueza y diversidad en la selva Amazónica.

- Al comparar este trabajo con otros realizados en la Amazonia se concluye que las enfermedades más comunes son las gastrointestinales y febrífugas, por lo que el uso y aplicación de diferentes plantas que alivien estas dolencias es compartido por las etnias del Trapecio. Sin embargo, la importancia de las plantas usadas para tratar estas dolencias depende de la cosmología y cosmogonía particular de cada comunidad.

- A partir del resultado de los índices de Fitoetnoendemicidad (IFEE), Fitoetnoaloctoneidad (IFEA), se concluye que Macedonia no tiene un nivel de aislamiento y si un alto grado de intercambio cultural a través del comercio. Por lo que la población no tiene necesidades biológicas o culturales distintas a las de otros grupos humanos en la amazonia (5).

- Es evidente el amplio interés que tienen empresas extranjeras y/o nacionales en conocer y usar a su beneficio personal el conocimiento tradicional y cultural que hay en la Amazonia, ya que su diversidad biológica la constituye como una fuente importante de químicos y material genético de valor comercial. Esto ha generado que los países ricos en biodiversidad no se beneficien del uso comercial del oro verde en sus territorios. Ejemplos claros de esta "Biopiratería" son las patentes de uso del Yajé, Copoazu, Curare, las cuales se realizaron a pesar del Convenio sobre la Biodiversidad Biológica ,1993(46) y la decisión 391 de 1996, en donde se legitimizó la importancia del origen del conocimiento y el reconocimiento de los beneficios asociados a los recursos genéticos y sus productos derivados. Por el incumplimiento de lo anterior, se han se han cometido "robos" a partir de los cuales se han justificado "genocidios culturales". Esto es un paradigma dentro de esta investigación, ya que como se plantean y hacen cumplir las políticas internas actuales en Colombia, la visión que existe entre la "soberanía étnica", producción en masa y el TLC, este tipo de conocimiento tradicional siempre quedara expuesto a la explotación occidental.

\section{Recomendaciones}

A partir de la constatación de las plantas en el herbario amazónico (COAH), se reportan plantas que no estaban catalogadas para la Amazonia, por lo que se podrían realizar estudios posteriores al por qué y cómo se introdujeron a esta parte del país. Así mismo, resulta pertinente para cualquier profesional que trabaje en comunidades indígenas ir en pro de la perpetuidad cultural, por lo que se deben generar proyectos que afiancen el conocimiento tradicional de cada una de las etnias indígenas, generando un sincretismo que una a la escuela con la Maloca, articulándola desde un proyecto educativo institucional (PEI).

\section{Agradecimientos}

Agradezco a Femando Quintana, Ana Luz Arias, y Andrés Quintana por el apoyo incondicional a lo largo de este estudio, a Isabel Buitrago por su amistad e incondicionalidad a lo largo del trabajo, asimismo a Juan Carlos Arias por su apoyo al momento de la entrada a la identificación de todas las muestras vegetales en el instituto de investigaciones científicas (SINCHI) y a lo largo del trabajo, a Germán Peńa del Águila y Familia por abrirme las puestas de su hogar, a la profesora Rosaura Miraña, al profesor Antero León Macedo, Javier Peña, Alfonso Peña, Inés León Macedo, Israel Bastos, al curaca Job Peńa y a todos los integrantes de la Comunidad indígena de Macedonia por apoyar la ejecución del estudio; a Daniel Fonseca (Subdirector de proyectos regionals del SINCHI) por la dirección del trabajo, aportes y revisión del manuscrito. 


\section{Referencias}

1. Moreno, E. 2005. Estudio etnobotánico de las plantas medicinales de san José de Suaita, Suaita (Santander). Tesis de grado (Biología). Universidad Nacional de Colombia. Bogotá.

2. Bermúdez, A. \& Velázquez, D. 2002. Etnobotánica medica de una comunidad campesina del estado Trujillo, Venezuela: Un estudio preliminar, usando técnicas cuantitativas. Revista de la facultad de farmacia, volumen \#44 Universidad Simón Bolívar Caracas, Venezuela.

3. Díaz, M. 1998. Estudio etnobotánico de las plantas empleadas por doña Romelia, Terapeuta Tradicional del Municipio de Mocoa (Putumayo). Tesis de grado (Biología) Pontificia Universidad Javeriana. Bogotá, Colombia.

4. Milliken, W \& Albert, B. 1997. The use of medicinal plants by the Yanomi Indians of Brazil, pat II. Economyc Botany. 50(3): 264-278.

5. Mesa, S. \& Jiménez, S. 1996. Algunos elementos para el análisis numérico de los datos en la etnobotánica. Universidad Complutense. Madrid, Espańa.

6. Mantilla, L; Cárdenas, D; Arias, J; Vanegas, J; Jiménez, D; Vargas, O; Gómez, Luís. 2007. Plantas útiles y promisorias en la Comunidad de Wacurabá (Caño Cuduyarí) en el departamento de Vaupés (Amazonía colombiana). Instituto Amazónico de Investigaciones Científicas.

7. Arias, J; Prieto, A. 2005. Diversidad Biológica del sur de la Amazonia Colombiana. Capítulo 2. Corpoamazonia. Leticia, Colombia

8. Gonzales, M. 1997. Catalogo preliminar comentado de la flora del medio Caquetá. Editorial. Tropembos Colombia.

9. Reichel-Dolmatoff, G. 1997. Chamanes de la Selva Pluvial, Themis Books. London, England.

10. Germosén-Robineau L. 1995. Hacia una farmacopea vegetal caribeña. Edición TRAMIL 7. Enda - Caribe, UAG \& Universidad de Antioquia. Santo Domingo. p. 696

11. Phillips, O. 1996. Some quantitative methods for analyzing ethnobotanical knowledge, the New York botanical garden.

12. Van der Hammen, M. 1992. El manejo del mundo, naturaleza y sociedad entre los Yukuna de la amazonia colombiana. Estudios en la amazonia Colombiana Tomo IV. Tropembos y tercer mundo editores. Bogotá, Colombia.

13. Shultes, R. \& Raffauf, R. 1990. The Healing Forrest. Medicinal and toxic plants of the northwest Amazonia. Dudley, T. (ed) Historical, Ethno-\& Economic Botany Series. Vol. 2. Dioscorides Press. Oregon.

14. Prance, G. Balee, W. Boom, M. Carneiro. R. (1987). Ethnobotany and the Case for Conservation in Amazonia. Conservation Biology, Vol. 1, No. 4 pp. 296-310.

15. Balee, W. 1986. Analise preliminar de inventario florestal e a etnobotánica Kảapor (Maranhao). Boletim do Museu Paraense Emilio Goeldi 2(2):141-167.

16. Palacios, P. 1986. Análisis de los usos y formas de manejo de algunas especies vegetales empleadas por las Comunidades Anoque, Huitoto y Miraña asentadas en la ribera del rio Caquetá. Colombia Amazónica, Vol. 2.

17. Glemboski, L. 1983. Ethnobotany of the Tikuna, Amazonas, Colombia. Instituto de Ciencias Naturales. Universidad Nacional de Colombia. Bogotá.
18. Declaración de Chiang Mai, 1998. "Salve vidas salvando plantas". Tailandia.

19. Carvajal Burbano, A (2009). Desarrollo y cultura. Elementos para la reflexión y la acción, 2a. Edición, Cali: Escuela de Trabajo Social y Desarrollo Humano-Universidad del Valle.

20. Zárate, C. (2001). Los condicionantes Sociales, Políticos e Institucionales del Ordenamiento Ambiental de la Amazonia. En T. R. Defler, \& P. A. Palacios, Zonificación Ambiental para el Ordenamiento territorial en la Amazonia colombiana (págs. 74-79). Leticia: Instituto Amazónico de Investigaciones (Imani).

21. Bejarano, M. 2003. Estudio de las plantas Medicinales utilizadas por la Comunidad indigena Camaritagua (Amazonas Colombia). Tesis de grado (Biología). Universidad Nacional de Colombia. Bogotá.

22. Palacios, P. 1986. Análisis de los usos y formas de manejo de algunas especies vegetales empleadas por las Comunidades Anoque, Huitoto y Miraña asentadas en la ribera del rio Caquetá. Colombia Amazónica, Vol. 2.

23. La Rotta, C. 1982. Estudio etnobotánico sobre las especies utilizadas por la Comunidad Indígena Miraña. Amazonas, Colombia. Fondo FEN. Manuscrito mecanografiado.

24. La Rotta, C. 1983. Observaciones etnobotánicas sobre algunas especies utilizadas por la comunidad indígena Andoque (Amazonas, Colombia). Tesis de grado (Biologia). Universidad Nacional de Colombia. Bogotá.

25. Riaño, E, 2003. Organizando su espacio, construyendo su territorio: transformaciones de los asentamientos TIKUNA en la ribera del Amazonas colombiano. Leticia: Universidad Nacional de Colombia, IMANI, Unibiblos.

26. Gasché, J. \& Echeverri J. 2003. "Sociodiversidad bosquesina: Un acercamiento desde el enfoque de una sociología comparativa". Proyecto IIAP-IMANI. Inédito.

27. Rahman, M.A. \& Fals, O, 1991. El punto de vista teórico de la IAP.Ed. Acción y conocimiento: Como romper el monopolio con la investigación-acción participativa. Bogotá, Colombia: Cinep pp 37-50.

28. Adu-Tutu. M, Afful. Y, Asante-Appiah. K, Leberman. D, Hall. J, Elvinlewis, M. 1979. Chewing Stick Usage in Southern Ghana. Econ Bot. p. 320-328.

29. Phillips, O. \& Gentry, A. 1993. The Useful Plants of Tambopata, Peru: I. Statistical Hypotheses Test with a New Quantitative Technique.

30. Moreno, E. 2005. Estudio etnobotánico de las plantas medicinales de san José de Suaita, Suaita (Santander). Tesis de grado (Biología). Universidad Nacional de Colombia. Bogotá.

31. Farnsworth, N; Akerele, O.; Bingel, A.; Soejarto, D. \& Guo, Z. 1985. Medicinal Plants in Therapy. Bull. of the World Health Org. 63(5): 965-981.

32. De los Ríos, C.; Hidalgo-Báez, D.; Contreras Q. \& Crescente, O. 1999. Phytochemical Evaluation and Antibacterial Activity of Espeletia schultzii (Asteraceae) Inflorescences. Ciencia. 7: 72-77.

33. Khafagi, I. \& Dewedar, A. 2000. The Efficiency of Random versus Ethnodirected Research in the Evaluation of Sinai Medicinal Plants for Bioactive Compounds. J. of Ethnopharm. 71: 365-376.

34. Germosén-Robineau, L. 1995. Hacia una farmacopea vegetal caribeña. Edición TRAMIL 7. Enda - Caribe, UAG \& Universidad de Antioquia. Santo Domingo. p. 696. 
35. Mesa, S. \& Jiménez, S. 1996. Algunos elementos para el análisis numérico de los datos en la etnobotánica. Universidad Complutense. Madrid, Espańa.

36. Cavalcante, P. \& Frikel, P. 1973. Farmacopéia Tiriyó. Estudo Etnobotânico. Publicação Avulsa. Museu Paraense Emílio Goeldi, Belém, Brasil. p. 24:145.

37. Boom, B. 1987. Los indios Chácobo y su Selva: Etnobotánica de una Tribu Panoan en el Noreste de Bolivia. En: Comunicación No. 7. La Paz, Bolivia. p. 25

38. Bennett, B. 1992. Plants and people of the Amazonian rain forest: the role of ethnobotany in sustainable development. BioScience. Vol. 42; p. 600-607.

39. Ullán de la Rosa, J. 2000. Los indios Tikuna del alto amazonas ante los procesos actuales de cambio cultural y globalización, Revista espańola de antropología americana \#30: 291-336.

40. Mello, M. 2002. Uso e diversidade de plantas de plantas medicinais por Cablocos do Baixo Amazonas, Barcerena, Pa, Brasil. Bol. Mus. Para. Emilio Goeldi, Ser. Bot. 4(1): 47-131.

41. Estrella, E. 1995a. Biodiversidad y salud en las poblaciones indígenas de la amazonia. Tratado de cooperación amazónica. Lima, Perú.
42. Quintana, R. 2009. El "verdadero" guardián del oro verde, estudio etnobotánico en la comunidad indígena Tikuna del alto amazonas, Macedonia. Tesis pregrado (Licenciatura en Biología). Universidad Distrital Francisco José de caldas. Bogotá, Colombia.

43. Díaz, M. 1998. Estudio etnobotánico de las plantas empleadas por doña Romelia, Terapeuta Tradicional del Municipio de Mocoa (Putumayo). Tesis de grado (Biología) Pontificia Universidad Javeriana. Bogotá, Colombia.

44. Zuluaga, G. \& Correa, C. 2002. Medicinas tradicionales: introducción al estudio de los sistemas tradicionales de salud y su relación con la medicina moderna. Editorial Kimpres. Bogotá, Colombia.

45. Zuluaga, G. 1999. Elementos de reflexión para la conservación de la Diversidad Biológica y cultural. "Taller sobre experiencias prácticas en gestión de Áreas Protegidas por los pueblos indigenas en Iberoamérica". Noviembre 29 a diciembre 3. Cartagena de Indias, Colombia.

46. ONU (Organización De Las Naciones Unidas). 1993. Convenio sobre la Biodiversidad biológica. Rio de Janeiro, Brasil.

47. Comisión del acuerdo de Cartagena, Decisión N. 391 Y 369: Régimen Común acceso a los recursos genéticos, Caracas, Julio 2 de 1996. 